\title{
Lyndal Roper, Martin Luther. Renegade and Prophet
}

\section{Gérald Chaix}

\section{OpenEdition \\ Journals}

Édition électronique

URL : http://journals.openedition.org/ifha/9170

DOI : 10.4000/ifha. 9170

ISSN : 2198-8943

Éditeur

IFRA - Institut franco-allemand (sciences historiques et sociales)

\section{Référence électronique}

Gérald Chaix, "Lyndal Roper, Martin Luther. Renegade and Prophet », Revue de I'IFHA [En ligne], Date de recension, mis en ligne le 20 juin 2018, consulté le 24 septembre 2020. URL : http:// journals.openedition.org/ifha/9170 ; DOI : https://doi.org/10.4000/ifha.9170

Ce document a été généré automatiquement le 24 septembre 2020.

(C)IFHA 


\title{
Lyndal Roper, Martin Luther. Renegade and Prophet
}

\author{
Gérald Chaix
}

\section{RÉFÉRENCE}

Lyndal Roper, Martin Luther. Renegade and Prophet, London: The Bodley Head, 2016, XIV-577 p., $30 £$

Sans aucun doute l'une des meilleures contributions de l'année Luther, comme en témoigne d'ailleurs la rapide traduction en allemand. Trois raisons à cela. Tout d'abord, la qualité de l'information qui résulte d'une lecture attentive des écrits de Luther, notamment de la correspondance et des propos de table, ainsi que d'une connaissance approfondie de la littérature secondaire. Ensuite, sans ignorer les analyses controversées d'Erikson (Luther avant Luther) et de Fromm (La Peur de la liberté), l'originalité de l'approche psychologique qui fait de l'analyse du développement intérieur de Luther l'objectif constant du livre. Enfin, ordonné en dix-neuf chapitres qui concilient récit chronologique et analyses thématiques, l'ouvrage est écrit de manière claire et d'une plume alerte, tandis que les illustrations, abondantes et finement commentées, étayent continûment la démonstration. Lyndal Roper entend également fournir une véritable histoire sociale de la Réformation, ni téléologique (en faisant de la Réformation le fondement de la démocratie) ni anachronique (en considérant Müntzer comme le prototype du révolutionnaire).

Le premier chapitre consacré à la famille de Luder et à l'activité à Mansfeld est à cet égard exemplaire. Il tire pleinement profit des récentes recherches archéologiques et archivistiques. Il confirme qu'à Mansfeld, ville minière, la famille Luder vivait confortablement, en dépit d'une insécurité permanente et d'une violence menaçante, et que le père de Martin ne manquait sans doute pas d'ambition pour son fils. Celle-ci contribue à expliquer l'investissement éducatif : les premières années à l'école latine de Mansfeld, un passage d'à peine un an à celle de Magdeburg (1497/8), quatre années à celle d'Eisenach, d'où la famille maternelle était originaire, jusqu'à l'immatriculation à 
l'université d'Erfurt en 1501. On comprend la déception paternelle lorsqu'à la veille d'entamer ses études de droit, à l'été 1505, Martin Luder décide de rentrer au couvent des Augustins d'Erfurt, renonçant ainsi et à la carrière à laquelle il était promis et au mariage auquel il était peut-être destiné. En signe de désapprobation, son père, Hans Luder, qui était passé par respect au voussoiement lorsque son fils était devenu maîtreès-arts, revient alors au tutoiement. Pour L. Roper, cette rébellion contre l'autorité paternelle est déterminante. Elle rappelle ainsi le propos de table dans lequel, une trentaine d'années plus tard, Luther évoquait sa juvénile révolte et l'interprétait comme le choix d'un monde matriarcal, peuplé de figures religieuses féminines et empreint d'une fausse religiosité. Un choix qui, note-t-elle, ne fut pas sans susciter à son tour des problèmes d'ordre spirituel. Elle éclaire sous ce jour les relations de Luther et de son confesseur Staupitz, revient sur la paternité spirituelle que celui-ci a exercée sur son jeune et talentueux confrère et souligne les différences qui distinguent cependant les deux hommes. L. Roper insiste également sur l'importance de l'enracinement saxon : celui d'un territoire princier, très différent du système politique prévalant dans les villes de l'Allemagne méridionale. Il n'est pas sans influence, selon elle, sur le « conservatisme » du réformateur.

Le récit du cheminement de Luther du couvent d'Erfurt (chapitre 3) jusqu'à la diète d'Augsbourg et à la rédaction de la Confession d'Augsbourg en 1530 (chapitre 15) est précis et détaillé. En octobre 1517, la publication des thèses « libère » personnellement Luther mais s'inscrit dans une démarche commune à l'ensemble de la faculté de théologie de Wittenberg, subjuguée par l'énergie du frère augustin et progressivement ralliée à ses convictions. L. Roper met en évidence l'importance des « disputes " (Heidelberg, Leipzig), des polémiques, des affrontements (Cajetan) et des condamnations (pontificale et impériale) dans l'évolution, régulièrement rendue publique, de la pensée de Luther au cours des deux années suivantes et notamment de l'année 1520 et dans son « engagement » (chapitre 7). Prêt au martyr, Luther manifeste lors de la diète de Worms, en 1521, face à Charles Quint, sa capacité à argumenter. Il affirme à cette occasion les droits de la « conscience » (Gewissen), c'est-à-dire de cette conviction intérieure qui résulte du travail de la raison et des exigences de la foi. Tandis que Luther, mis au ban de l'Empire, est caché durant dix mois au château de la Wartburg, où il déploie une intense activité, les événements s'accélèrent. Ils obligent Luther, revenu à Wittenberg, à prendre position. Il s'oppose à Karlstadt et adopte le point de vue, temporisateur, des autorités princières et communales. C'est ce même point de vue qui l'oppose à nouveau, en 1524, à Karlstadt et à Müntzer, qu'il range dans la catégorie des Schwärmer, et, plus largement, aux mouvements séditieux des paysans, qu'il condamne vigoureusement. Persuadé de l'origine diabolique des troubles qui mettent en péril l'ordre social et rappellent l'imminence de la fin des temps, Luther accumule les gestes de rupture. Il abandonne son froc d'augustin (octobre 1524). Ne reconnaissant aucune valeur au célibat, il décide à son tour de se marier au printemps 1525, peu de temps après la mort de l'Électeur Frédéric (5 mai 1525) et de celle de Staupitz (28 décembre 1524), comme si la disparition de son père spirituel lui avait enfin permis d'endosser à son tour la paternité. Il affranchit la sexualité de tout lien avec le péché, exalte les joies de la vie conjugale et familiale, sans pour autant mettre en question l'« ordre naturel » qui fonde, pour lui comme pour ses contemporains, l'autorité maritale et paternelle.

C'est aussi le moment où il rompt ostensiblement avec Érasme, tandis qu'il continue de s'opposer à Karlstadt sur la signification de l'eucharistie. La question de la présence 
réelle divise profondément les théologiens. Dans le sud de l'Allemagne, nombre d'entre eux rallient les positions de Zwingli, le réformateur de Zurich, pour qui les paroles " ceci est mon corps » ne doivent pas être comprises littéralement. Pour Luther, ce combat est directement dirigé contre le diable. Ce même diable qui n'hésite pas à l'assaillir et à lui faire perdre connaissance le 6 juillet 1527. Mais la peste qui frappe Wittenberg un mois plus tard épargne Luther qui a refusé de fuir. C'est le signe qu'il a raison. Il défend sa position lors du colloque de Marburg en 1529. Souhaité par le landgrave de Hesse, l'accord échoue. La dynamique est de plus en plus locale et territoriale. Une nouvelle Église se met ainsi en place en Saxe électorale. À la Diète qui se tient à Augsbourg en 1530, en l'absence de Luther toujours banni, une profession de foi (Confessio Augustana), rédigée pour l'essentiel par Melanchthon, est remise à Charles Quint, qui la rejette. La fracture confessionnelle se précise.

Après 1530, le récit s'accélère. Pour L. Roper, Luther est devenu moins agile, tant physiquement qu'intellectuellement. C'est le temps de la « consolidation » des camps. Ils ont pour adversaires communs les « anabaptistes ». Animée par la Saxe électorale et le Hesse, la ligue de Smalkalde se constitue. Les juristes élaborent un « droit de résistance » face à l'empereur. De leur côté, les théologiens continuent de rechercher un terrain d'entente à propos de l'eucharistie. Alors que la papauté tarde à réunir le concile promis par l'empereur, celui-ci tente d'organiser des colloques religieux entre théologiens des deux confessions. Le premier a lieu à Regensburg (Ratisbonne), en marge de la Diète qui s'y réunit en 1541. L'irénisme de Melanchthon fait de lui l'interlocuteur privilégié. La santé de Luther devient de plus en plus chancelante. Les tensions se durcissent entre amis et ennemis du réformateur. Parmi ces derniers, le pape arrive en tête. Jusqu'au bout Luther en fait l'instrument du diable. Son attitude à l'égard des Turcs est plus contrastée. Il refuse l'idée de croisade contre eux, tout en justifiant la guerre menée contre leur politique d'expansion. Certes, il rejette le Coran, condamne leurs mœurs et notamment leur conception du mariage, mais développe les modalités concernant un véritable modèle de coexistence, à en croire L. Roper. En revanche, d'abord conciliant, en 1523, le discours à l'égard des Juifs devient franchement hostile à partir de 1543. Il est partisan d'une « complète éradication culturelle ». Conscient de son mauvais état de santé, Luther se rend néanmoins en Thuringe pour tenter une conciliation entre les comtes de Mansfeld qu'opposent choix confessionnels et conflits d'héritage. Il meurt au cours de ce voyage, à Eisleben, dans la nuit du 17 au 18 février 1546. L'exemplarité de cette mort est immédiatement soulignée par Justus Jonas, présent sur place. Elle est réaffirmée lors des funérailles à Wittenberg par Bugenhagen et Melanchthon.

Les conclusions de L. Roper sont claires. Religieusement, Luther est à l'origine d'une nouvelle Église. Politiquement, il donne naissance à la théorie des deux règnes qui distingue royaume de Dieu et royaume terrestre. Pour L. Roper, cette distinction ouvre la voie à une obéissance sans frein. Culturellement, Luther lègue à l'Allemagne entière sa traduction de la Bible qui marque profondément la langue allemande.

Théologiquement, il maintient la croyance en la présence réelle, surmonte l'opposition entre chair et esprit grâce à la vision positive qu'il a du corps. Profondément croyant, il reste soumis au doute, comme le rappelle Melanchthon dans la vie qu'il écrit sur son ami en 1555. Mais cette personnalité nous est devenue passablement étrangère aujourd'hui : son agressivité, son attrait pour la scatologie, son autoritarisme, son goût pour la démonisation de ses adversaires, son antisémitisme et son penchant pour la simplification en font un homme d'un autre temps. Des défauts 
qui expliquent en revanche le courage avec lequel il s'est attaqué aux obstacles qui s'opposaient à la réforme. Pour L. Roper, il faut donc cesser d'en faire l'initiateur de la « modernité », de la « liberté individuelle », de la « confessionnalisation », associant identité religieuse et identité politique, et se garder d'oublier sa salutaire défiance à l'égard de la raison, qu'il qualifie de " putain ».

INDEX

Index chronologique : Période moderne

Thèmes : Histoire religieuse ; Histoire des mentalités

\section{AUTEURS}

\section{GÉRALD CHAIX}

Université de Tours, UMR 7323 